\title{
INTRODUCTION
}

\section{Elucidating the causes and examining the latest clinical findings in pulmonary fibrosis}

\author{
T.E. King Jr* and R.M. du Bois ${ }^{\#}$
}

I diopathic pulmonary fibrosis (IPF) is a devastating disease, with a 5-yr survival rate of only $20-30 \%$ following diagnosis [1]. Patients with IPF may experience a rapidly progressive decline in lung function from onset or a slowly progressive course of illness, during which the development of acute exacerbations can lead to rapid respiratory failure and early death [2]. Prognosis is extremely poor and no pharmacological agent improves survival in IPF. Immunosuppressive treatment regimens have been used in the treatment of IPF; however, there is little evidence to support their efficacy and safety. There has been growing interest in the diagnosis and prevention of acute exacerbations in patients with IPF [3]. It has been shown that acute exacerbations are quite prevalent among patients with IPF, that they occur at any stage of the illness and that they are frequently fatal events. Consequently, this has created additional concern that treatment should be initiated at the earliest sign of impairment.

The present issue of European Respiratory Review reports the proceedings of an International Scientific Advisory Board Meeting on "Elucidating the causes and examining the latest clinical findings in pulmonary fibrosis", which was held in London (UK), on November 16-18, 2007. The first two articles explore the current understanding in pulmonary fibrosis and are followed by six articles that analyse the mechanisms of disease in IPF and challenge the once prevalent view of IPF as a disease of unremitting, chronic inflammation. The final article focuses on the potential genetic associations with the disease.

\section{CLINICAL FINDINGS AND TREATMENT OF IPF}

In the first article, the authors discuss the advances in the classification of the idiopathic interstitial pneumonias (IIPs), in which IPF and its corresponding histopathological pattern of usual interstitial pneumonia (UIP) are distinguished from six non-IPF (IIP) subtypes [4]. This distinction is important because IPF has a uniquely devastating prognosis compared with the other IIPs. The authors highlight important advances in the diagnosis of IPF, including the role of high-resolution computed tomography (HRCT) as a diagnostic tool without recourse to biopsy. The article emphasises the importance of an interactive clinicalradiographical-pathological approach to the diagnosis of the IIPs and recommends that, wherever possible, patients should be referred to centres with expertise in diffuse parenchymal lung disorders in order to help clarify the diagnosis and provide suggestions regarding treatment options.

IPF is among the most therapeutically challenging lung disorders. In the second article, BROWN and WELLS [5] discuss the lessons that have been learnt from recent clinical trials of potential new therapies for IPF. Conventional approaches to the treatment of IPF have been largely empirical and were based on the belief that IPF was a chronic inflammatory disease. More recently, following the emergence of a better understanding of underlying disease mechanisms, treatment approaches have been based on targeting the molecular events believed to perpetuate and sustain the fibrotic process in IPF. The authors reviewed clinical trials of potential new IPF therapies. Few of the studies met their primary end-points. In many cases, there were marginal trends in favour of the primary endpoints or, most often, there were statistically significant trends found for one or more exploratory end-point. For example, in the randomised, placebo-controlled Bosentan Use in Interstitial Lung Disease (BUILD)-1 trial, which they discuss in detail, a trend in favour of bosentan was observed in the secondary end-point of time-todisease progression or death at 12 months [6]. Post hoc analysis revealed a more pronounced treatment effect in favour of bosentan in the subset of patients who underwent surgical lung biopsy to confirm diagnosis of IPF [6]. These secondary and post hoc analyses will be tested in the ongoing BUILD-3 study of bosentan. BROWN and WELLS [5] emphasise the need for confident diagnoses of IPF if clinical trial results are to be meaningful and highlight the need for validated outcome measures that represent either improvement or disease progression.

\section{PATHOGENESIS OF IPF}

The following six articles in the present issue discuss our current understanding of the

\section{AFFILIATIONS}

*Dept of Medicine, University of California San Francisco, San Francisco, CA, and

\#Dept of Medicine, National Jewish Health, Denver, CO, USA.

CORRESPONDENCE

T.E. King Jr

Dept of Medicine

University of California San Francisco (UCSF)

505 Parnassus Avenue

Box 0120

San Francisco

CA 94143-0120

USA

Fax: 14155025869

E-mail: tking@medicine.ucsf.edu

\section{STATEMENT OF INTEREST}

T.E. King Jr has served on advisory boards for Actelion, InterMune, GlaxoSmithKline and ImmuneWorks, and has also served as a consultant for Nektar, Alexza, AstraZeneca, Biogen, Centocor, Fibrogen, Genzyme, Human Genome Sciences, Merck and CoTherix. R.M. du Bois has received travel grants and is/has been a paid consultant, Steering Committee member and/or Co-Chair of a Steering Committee in the past 5 yrs for Intermune, Actelion, Centocor, Boehringer Ingelheim, Novartis, Genzyme and MondoBiotech. 
pathogenesis of IPF. CHAMBERS [7] explores how abnormal wound repair may occur in IPF and suggests that abnormalities in several pathways involved in normal tissue repair may be involved. CHAMBERS [7] first discusses the differentiation and control of myofibroblasts, which are the key effector cells in pulmonary fibrosis and the resulting extracellular matrix (ECM) deposition. Secondly, the role of the coagulation system and its signalling molecules and receptors is discussed. Finally, the potentially critical role proteinase activated receptors (PARs) may play in the tissue injury and repair responses in the lung are discussed.

NobLE [8] provides an overview of the "epithelial injury" hypothesis. In the 1970s and 1980s, the prevailing view was that IPF was due to unremitting, chronic inflammation. The current epithelial injury hypothesis contends that pulmonary fibrosis arises from repeated cycles of alveolar epithelial cell (AEC) injury and the triggering of epithelial apoptosis, which leads to a process that mirrors abnormal wound healing [9]. Instead of AECs healing by repair, myofibroblast proliferation and extracellular matrix deposition continues unabated in IPF. In addition, NOBLE [8] discusses the process known as epithelialmesenchymal transition, the transformation of epithelial cells into mesenchymal cells, as a possible explanation for the activation of AECs in IPF.

Growing circumstantial evidence suggests that in IPF the alveolar epithelium is prone to apoptosis, "programmed" or "gene-directed" cell death [10, 11]. Conversely, there is recent evidence to suggest that decreased apoptosis of myofibroblasts, particularly in areas of collagen deposition, is also present in IPF [12]. UHAL [13] explores the concept of altered apoptosis in IPF and raises the intriguing, albeit theoretical, proposition that blocking epithelial cell apoptosis combined with the induction of myofibroblast apoptosis might be expected to resolve ongoing pulmonary fibrosis in IPF. Certainly, therapeutic intervention aimed at modifying apoptosis in IPF, and in turn fibrosis, is an intriguing prospect for future research.

The potent pro-fibrotic, pro-inflammatory vasoconstrictor endothelin (ET)-1 is emerging as an important candidate in the aetiology of IPF [14]. As data from the BUILD-1 trial suggests, drugs that target ET-1 may be of potential benefit in patients with IPF [6]. ET-1 provides the focus for the article by ABRAHAM [15], which examines recent evidence for the role of ET-1 in pulmonary fibrosis and, in particular, how it influences myofibroblast formation and function. Available data are primarily from in vitro studies but nevertheless point to the importance of ET-1 in the regulation of cell proliferation and differentiation, as well as in the deposition and turnover of ECM. For example, the addition of ET-1 to normal lung fibroblasts has been found to promote synthesis of collagen types I and III, by a mechanism dependent upon both ET-1 receptor subtypes $\mathrm{ET}_{\mathrm{A}}$ and $\mathrm{ET}_{\mathrm{B}}$ [16], while $\mathrm{ET}-1$ has also been shown to play a role in promoting fibroblast differentiation to a myofibroblast phenotype [17].

The article by KEANE [18] explores the role of chemokines and cytokines, key components of T-helper cell (Th)1- and Th2mediated immune responses, in pulmonary fibrosis. Although inflammation is known to be important in other pulmonary fibrotic diseases, unremitting, chronic inflammation is no longer viewed as the primary pathophysiological mechanism in IPF. However, KEANE [18] argues that although overt inflammation is usually absent by the time patients present with IPF, inflammation may still be involved in IPF. KEANE [18] hypothesises that with each episode of epithelial injury, in disease characterised by repeated epithelial cell injury followed by a progressive fibrotic response, an acute inflammatory response results in a release of early response cytokines in the local environment. If instead of a "normal" Th1-type mediated response, a polarised Th2 response ensues, this would favour a fibroproliferative reaction and the progressive scarring and fibrosis seen in IPF. KeANE [18] also presents evidence to suggest that vascular remodelling and angiogenesis may be associated with the development of fibrosis.

The article by SHEPPARD [19] discusses the role of integrins in pulmonary fibrosis. Integrins act as signalling proteins and are involved in virtually every aspect of epithelial cell behaviour; increasingly, evidence is emerging to suggest that they may also be implicated in some pathological conditions including pulmonary fibrosis [20]. SHEPPARD [19] presents data, predominantly from murine models of pulmonary fibrosis, which show that the integrin $\alpha \mathrm{V} \beta 6$ (highly induced in UIP) plays a critical role in the pathological activity of transforming growth factor (TGF)- $\beta$ in pulmonary fibrosis. The investigation of TGF- $\beta$ as a therapeutic target is fraught with difficulty because of its diverse homeostatic role [21]; however, inhibition of $\alpha \mathrm{V} \beta 6$ may offer the prospect of targeted, local inhibition of TGF- $\beta$ at sites of $\alpha \mathrm{V} \beta 6$ upregulation, such as in lung tissue.

\section{GENETICS OF LUNG FIBROSIS}

It is already known that familial IPF occurs as an autosomal dominant disorder with variable penetrance [22]. To date, only three candidate genetic mutations have emerged from studies of pulmonary fibrosis in affected families. The evidence for genetic factors in sporadic IPF is much less compelling. LOYD [23] explores mutations in the genes encoding surfactant protein $C$ (SFTPC) and components of the telomerase gene, i.e. hTERT and TERC. Further research in this field will surely reveal other important candidates and will one day help to identify future therapeutic targets for this hugely challenging disease.

This issue of European Respiratory Review is intended to provide pulmonologists, radiologists, thoracic surgeons, pathologists and scientists involved in interstitial lung diseases with insights into the classification and diagnosis of idiopathic pulmonary fibrosis, as well as provide current and evolving concepts of pathogenesis that will hopefully lead to new approaches to treatment. As all the authors suggest, the paradigms that define idiopathic pulmonary fibrosis have shifted significantly following important basic and clinical research. They also suggest that there is exciting evolution in the current understanding of individual patterns of disease and the pathological mechanisms and predispositions that underpin the fibrosing lung diseases, particularly idiopathic pulmonary fibrosis. Together these approaches will continue to uncover further important aspects of this devastating disease, which will hopefully lead to better therapies.

\section{ACKNOWLEDGEMENTS}

The authors would like to thank A. Gray for his role in the literature review and preparation of this manuscript. 


\section{REFERENCES}

$1 \mathrm{du}$ Bois R, King TE Jr. Clinical advances in the diagnosis and therapy of the interstitial pneumonias. Thorax 2007; 62: 1008-1012.

2 Martinez FJ, Safrin S, Weycker D, et al. The clinical course of patients with idiopathic pulmonary fibrosis. Ann Intern Med 2005; 142: 963-996.

3 Collard HR, Moore BB, Flaherty KR, et al. Acute exacerbations of idiopathic pulmonary fibrosis. Am J Respir Crit Care Med 2007; 176: 636-643.

4 Raghu G, Nicholson AG, Lynch D. The classification, natural history and radiological/histological appearance of idiopathic pulmonary fibrosis and the other idiopathic interstitial pneumonias. Eur Respir Rev 2008; 17: 108-115.

5 Brown KK, Wells AU. Recent clinical trials in idiopathic pulmonary fibrosis and the BUILD-1 study. Eur Respir Rev 2008; 17: 116-122.

6 King TE Jr, Behr J, Brown KK, du Bois RM, Raghu G. A randomized placebo-controlled trial of bosentan in patients with idiopathic pulmonary fibrosis. Am J Respir Crit Care Med 2007; 176: 1-7.

7 Chambers RC. Abnormal wound healing responses in pulmonary fibrosis: focus on coagulation signalling. Eur Respir Rev 2008; 17: 130-137.

8 Noble PW. Epithelial fibroblast triggering and interactions in pulmonary fibrosis. Eur Respir Rev 2008; 17: 123-129.

9 Selman M, King TE, Pardo A. Idiopathic pulmonary fibrosis: prevailing and evolving hypotheses about its pathogenesis and implications for therapy. Ann Intern Med 2001; 134: 136-151.

10 Plataki M, Koutsopoulos AV, Darivianaki K, Delides G, Siafakas NM, Bouros D. Expression of apoptotic and antiapoptotic markers in epithelial cells in idiopathic pulmonary fibrosis. Chest 2005; 127: 266-274.

11 Uhal BD, Gidea C, Bargout R, et al. Captopril inhibits apoptosis in human lung epithelial cells: a potential antifibrotic mechanism. Am J Physiol 1998; 275: L1013-L1017.
12 Moodley YP, Caterina P, Scaffidi AK, et al. Comparison of the morphological and biochemical changes in normal human lung fibroblasts and fibroblasts derived from lungs of patients with idiopathic pulmonary fibrosis during FasL-induced apoptosis. J Pathol 2004; 202: 486-495.

13 Uhal BD. The role of apoptosis in pulmonary fibrosis. Eur Respir Rev 2008; 17: 138-144.

14 Giaid A, Michel RP, Stewart DJ, Sheppard M, Corrin B, Hamid Q. Expression of endothelin-1 in lungs of patients with cryptogenic fibrosing alveolitis. Lancet 1993; 341: 1550-1554.

15 Abraham D. Role of endothelin in lung fibrosis. Eur Respir Rev 2008; 17: 145-150.

16 Shi-Wen X, Denton CP, Dashwood MR, Holmes AM, BouGharios G, Pearson JD, et al. Fibroblast matrix gene expression and connective tissue remodeling: role of endothelin-1. J Invest Dermatol 2001; 116: 417-425.

17 Shi-Wen X, Chen Y, Denton CP, et al. Endothelin-1 promotes myofibroblast induction through the ETA receptor via a rac/phosphoinositide 3-kinase/Akt-dependent pathway and is essential for the enhanced contractile phenotype of fibrotic fibroblasts. Mol Biol Cell 2004; 15: 2707-2719.

18 Keane MP. The role of chemokines and cytokines in lung fibrosis. Eur Respir Rev 2008; 17: 151-156.

19 Sheppard D. The role of integrins in pulmonary fibrosis. Eur Respir Rev 2008; 17: 157-162.

20 Horan GS, Wood S, Ona V, et al. Partial inhibition of integrin $\alpha v \beta 6$ prevents pulmonary fibrosis without exacerbating inflammation. Am J Respir Crit Care Med 2008; 177: 56-65.

21 McCartney-Francis NL, Frazier-Jessen M, Wahl SM. TGF- $\beta$ : a balancing act. Int Rev Immunol 1998; 16: 553-580.

22 Steele MP, Speer MC, Loyd JE, et al. Clinical and pathologic features of familial interstitial pneumonia. Am J Respir Crit Care Med 2005; 172: 1146-1152.

23 Loyd JE. Gene expression profiling: can we identify the right target genes? Eur Respir Rev 2008; 17: 163-167. 